\title{
Grain Insect Monitoring Based on Imaging Sensor and Wireless Sensor Networks
}

\author{
Xiaoyu DU, Xiong SHI \& Lin LI \\ Wuhan Polytechnic University, Electrical and Electronic Engineering Institute, Wuhan, 430023, China \\ *Corresponding author
}

\begin{abstract}
Imaging sensor and wireless sensor networks are combined in this essay to establish the wireless grain insect monitoring network. The micro camera constituted of CMOS imagining sensor embeds the built-in nodes in a specific location in bulk grain. The light and bait of LED built in the nodes can attract the insects to go into the photographing region. The camera shoots the insects on the instruction of control center, then the wireless transmission module of application integrated in CPU transmits the photograph to the computer as of control center. In this way the information of grain insects in bulk grain are collected as reliable foundation for the prevention and cure of grain insects.
\end{abstract}

KEYWORD: grain insect monitoring, CMOS imaging sensor, wireless sensor networks

\section{GENERAL INSTRUCTIONS}

The prevention on grain insects in the process of grain bulk storage is first to detect the insects, then according to the species and quantity of the insects to decide the countermeasures. The monitoring in this course is much more complex than other single physical quantity monitoring. Because the insects' appearance and color are the main judgment evidences for insects identification and classification, in other words, only by observing the insects or their images, the accurate judgment can be made [1]. It is obvious that by the imaging sensor can make the insects monitoring more visualized and convenient to get more information.

The micro camera constituted of CMOS imaging sensor as core, and system single-chip of integrated wireless transmission module as control center, wireless transmission network node is constituted with traps, LED, lithium battery and charge management module, etc. This network node is placed in a specific location in the bulk grain or the location where the insect pest is most likely to occur, usually in several meters deep. The light of LED and traps attract the insects go into the photographing region of the camera. The camera shoots automatically or by instructions of control center. The pictures are transmitted by wireless transmission to forwarding node and finally to the computer as of control center. The collection and monitoring for grain insects information in bulk grain are achieved by the indication and following processing.

\section{SYSTEM STRUCTURE AND OPERATING PRINCIPLE}

Usually there are several storehouses in grain depot, and each can store tens or hundreds of tons. According to the Grain and Oil Storage Specification: monitoring points should be set at four corners in each room in the storehouse, one meter deep in the grain, and for the silo or squat silo the monitoring points are set nearby the wall at east, west, north and south. In addition that by the insects' habit monitoring points should be set where the insects easily appear. So the network node with micro camera should be embedded at one meter deep, and considering the blocking and limited battery capacity, a forwarding node will be set in each storehouse. The whole structure of monitoring network is as figure 1 .

The monitoring network is constituted of photograph sampling node in grain, forwarding node in each storehouse (main node) and the computer [2]. The forwarding nodes accept the photograph from each network node in the storehouse and transmit to the computer which is the destination.

The information transmission between forwarding node and computer can be network transmission, serial transmission or other. Being wired or wireless is determined on the basis of the scale and layout of the storehouse. 


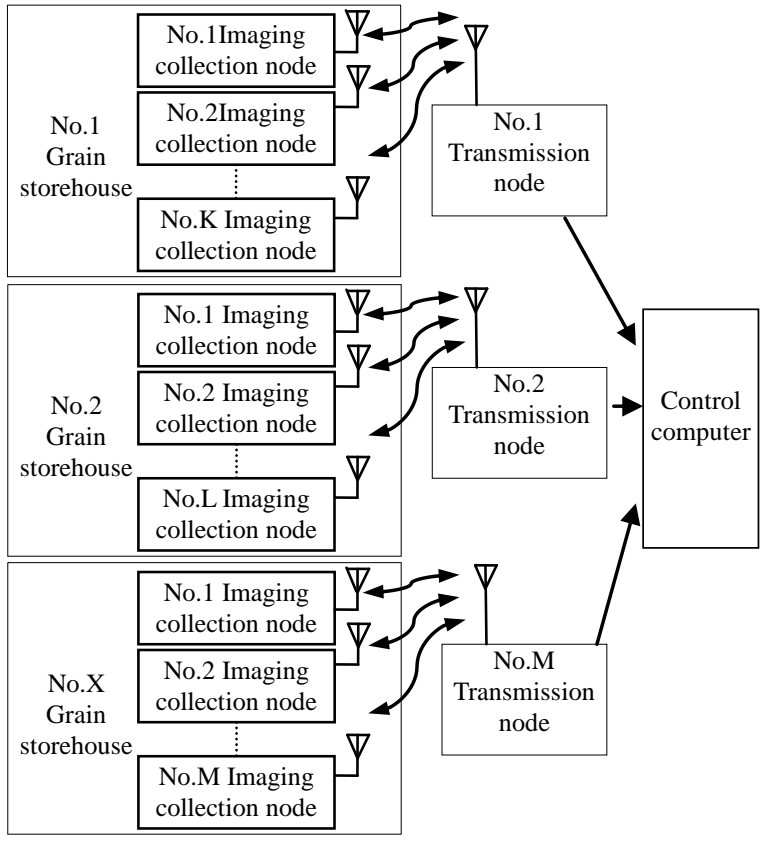

Figure 1 Structure of the grain insect monitoring network system

The computer is not only the control center, but also is the destination of all photographs. Considering that the quantity of storehouse is limited and quantity of the network nodes is less than ten in each house, meanwhile the time is enough in photograph transmission at wide intervals, roll-call polling is used in network transmission course [3].

All the nodes are numbered in order and the computer send inquiry instruction to each node in turn. The node shoots the photographing region when receives the instruction, and then unload the photograph by wireless communication channels. The computer will inquire next node after receive the grain insect photograph of this node. Monitoring software can set different priority for the monitoring points at different location. High priority can be given to the region where insects easily occur and more inquiry can be added.

There is clock calendar chip in network nodes, and the system can set shooting time for automatic shooting and uploading. Without doubt simultaneous transmission of two or more nodes should be prevented to avoid conflict in wireless transmission channels.

\section{DESIGNS OF THE NODES}

\subsection{Construction of imaging collection nodes}

Usually fumigation is used in deinsectization and the potion is highly toxic and caustic, so wired monitoring is easy to be damaged. Wireless sensor network can avoid this, but the node of network should be able to resist corrosion. The shell of the node must be sealed and corrosion-resistant.

The shape of imaging collection node is spherical with $40 \mathrm{~mm}$ diameter as a Ping-Pong ball. The shell can be wholly transparent and also can be transparent for hemisphere where the insects photographing are taken. In photographing region there are small holes for insects to go into the focal region in the shell; in this hemisphere a transparent sealer is added to protect the camera, LED, the printed circuit board and other electronic pieces. Structure of node is as figure 2.

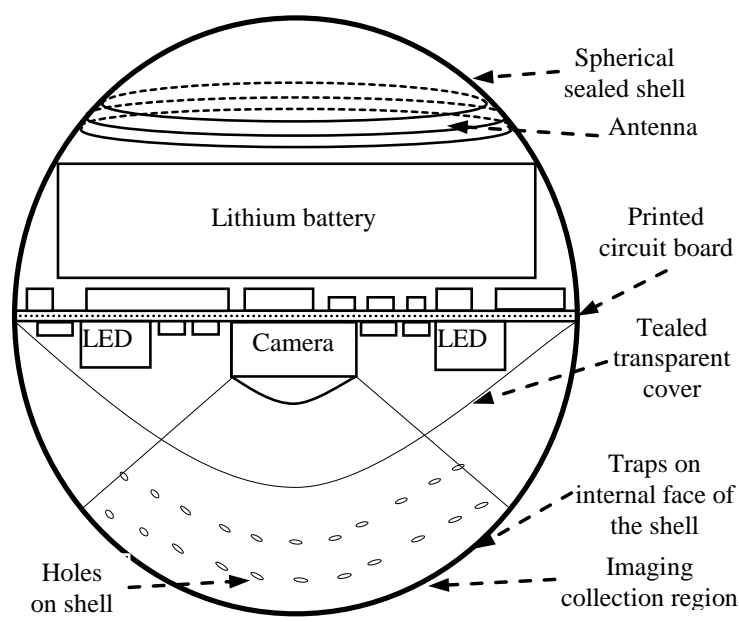

Figure 2 Structure of imaging collection node

\subsection{Circuit composing of imaging collection nodes}

The circuits of imaging collection nodes include CMOS camera, buffer store, single chip with integrated RF transceiver, lithium battery, wireless charging circuit, LED circuit and so on. Structure of composing circuit is as figure 3 .

From vacuum tube photoelectric imaging sensor, to charge-coupled device (CCD) imaging sensor, to CMOS imaging sensor, the imaging sensor has rapid development in decade with the improvement of electronic technologies. The micro camera constituted of CMOS imaging sensor in this essay, with small volume and low power consumption, can output photograph in multiple formats and high resolution. Considering the cost, camera with low pixel, as OV9655, can be chosen. Imaging sensor with 5 mega-pixel or much higher pixel, for example OV5640, can be used when high resolution image are in need. The images are sent to single chip through buffer store, and then dispatched to the main node by the wireless transmission module inside the chip.

Taking the camera with OV9655 imaging sensor chip as example, it can output 1.3 million pixel image, and the resolution can reach $1280 * 1024$. The image formats are YUV/YCbCr, GRB, GRB565, GRB555 and Raw GRB [4]. Figure 4 is the insect image this camera shot.

Pixel array, sweep circuit, A/D controller and other circuits are integrated in CMOS. Its core mission is to output continuous image sequence, and when photographing the program on photographing control function needs to write individually on de- 
mand. Taking OV5640[5] as example, the procedure which controls it to achieve photographing is shown in Figure 5.

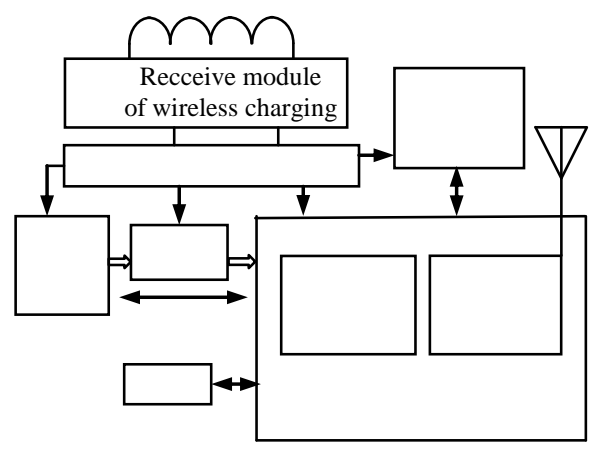

Figure 3 Diagram of circuit composing of imaging collection nodes

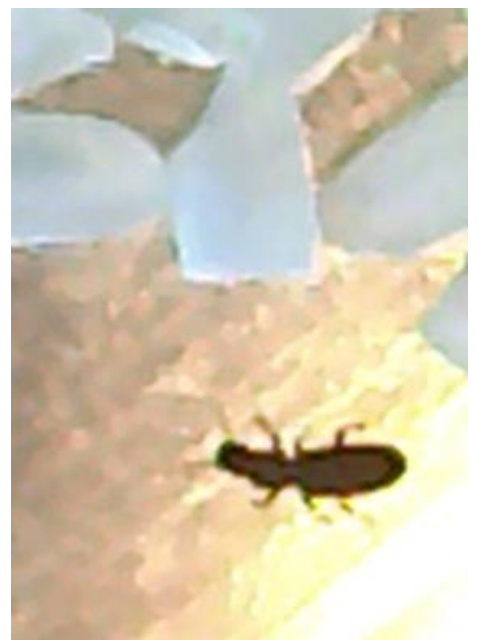

Figure 4 The insect image of camera shot

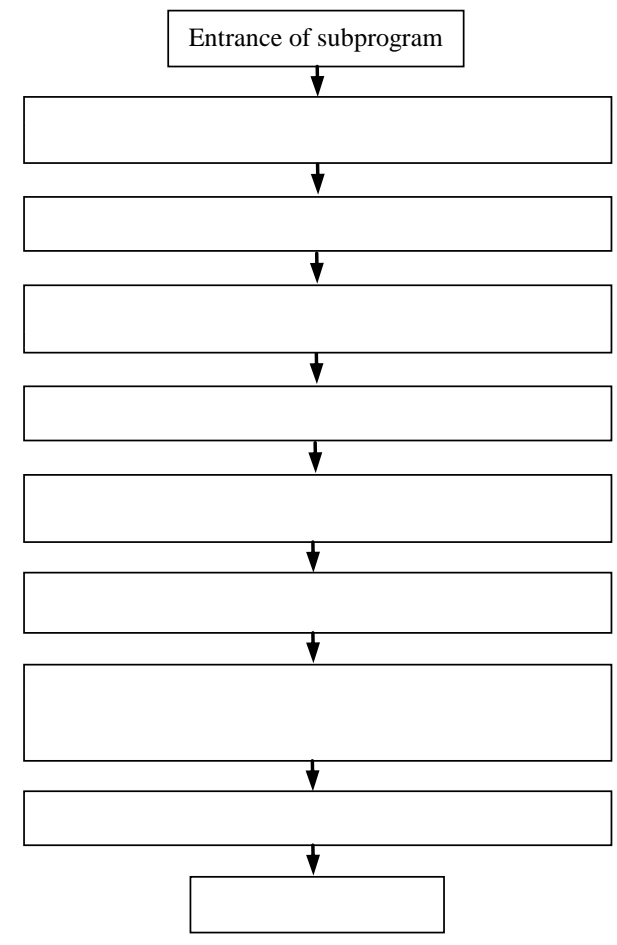

Figure 5 Diagram of OV5640 photographing procedure

When outputting the image with highest resolution, the camera with 1.3 million pixel will export 1 megabyte document and with 5 mega-pixels can get multi-megabyte document. If the speed to output the data is $20 \mathrm{~Kb} / \mathrm{s}$, it will spend ten second or hundreds seconds to send one image. The development and growth of grain insects is a long course, therefore in the Grain and Oil Storage Specification, only one or two sampling is needed each month. Real-time collection and transmission are not necessary in monitoring, so low transmission rate is enough.

The node should be sealed for corrosion protection, so its battery cannot be replaced and charging socket cannot be used. The TI bqTESLA wireless charging module is set. Post the matched charging socket after recycling the network node, which is similar to the way to charging electric toothbrush. Certainly sleeping mechanism should be used in node to reach low power consumption, in order to extend service life.

The node for grain insects monitoring should be embedded in grain, so LED is installed nearby the camera to provide lighting, which is controlled by CPU. The LED can also work as insects trap. There are many holes in imaging collection region which on the shell of collection node. Making use of the photokinesis of insects, the light and traps can attract insects to go into photographing region.

The wireless single chip Si1060 in Silicon Labs, which integrates the controller with high speed and low power consumption and wireless RF transceiver module, can be used as controller and RF module ${ }^{[6]}$.

\subsection{Design of transmission nodes}

The transmission nodes receive the data from imaging collection nodes in the storehouse and transmit to the computer, at the same time transmit the instruction of computer to each node. The received data are temporarily saved in extended memory. The information transmission between transmission nodes and computer can be wired or wireless serial communication or network communication, on the basis of distribution of the storehouses.

In transmission node, wireless single chip Si1060 is also used. Only by building a simple circuit around, can the wireless receive and dispatch be realized. Structure of circuit of transmission nodes is shown in figure 6 .

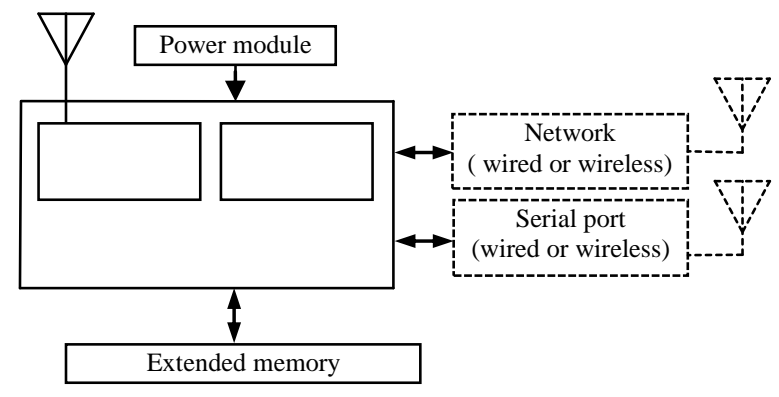

Figure 6 Circuit structure of transmission nodes 


\section{CONCLUSION}

Combining CMOS Imaging Sensor and wireless sensor networks, the wireless grain insects monitoring networks can directly monitor the situation of the grain insects in storehouse. It provides accurate data for grain pest control and reduces the loss caused by insect pest. China is not only a country with great grain industry, but also with big grain storage, so the wireless grain insects monitoring network has vast development prospect and application space in insects prevention for grain bulk storage.

\section{REFERENCES}

[1] Xuguang Bai, 2008, Storage Pest and Its Prevention, Beijing: Science Press.

[2] Li Tan, 2011, Wireless Sensor Network Theory and Technology, Beijing: Machine Press.

[3] Ming Chen, Bing Li, YongxingJia, 2004, Computer Networks, Beijing: Posts and Telecom Press.

[4] OmniVision Technologies. 2004, OV9650 Datasheet. USA: OmniVision Technologies.

[5] OmniVision Technologies. 2011.5, OV5640 Datasheet. USA: Omni Vision Technologies.

[6] Silicon Labs, 2014.2, Si106x/108x Datasheet. USA: Silicon Labs.

[7] This essay is subsidized by scientific research project in Wuhan Polytechnic University. 